\title{
Strong decay widths and coupling constants of recent charm meson states
}

\author{
Meenakshi Batra ${ }^{\mathrm{a}}$, Alka Upadhayay ${ }^{\mathrm{b}}$ \\ School of Physics and Material Science, Thapar University, Patiala 147004, India
}

Received: 22 January 2015 / Accepted: 10 June 2015 / Published online: 8 July 2015

(C) The Author(s) 2015. This article is published with open access at Springerlink.com

\begin{abstract}
Open charm hadrons with strange and nonstrange mesons have been discovered in recent years. We study the spectra of several newly observed resonances by different collaborations like BaBar (del Amo Sanchez et al., Phys Rev D 82:111101, 2010) and LHCb (Aaij et al. [LHCb Collaboration], J High Energy Phys 1309:145, 2013) etc. Using an effective Lagrangian approach based on heavy quark symmetry and chiral dynamics, we explore the strong decay widths and branching ratios of various resonances and suggest their $J^{p}$ values. We try to fit the experimental data to find the coupling constants involved in the strong decays through pseudo-scalar mesons. The present work also discusses the possible spin-parity assignments of recently observed states by the LHCb Collaboration. The tentative assignment of the newly discovered state $D_{J}^{*}(3000)$ can be by natural parity states $\left(0^{+}, 1^{-}, 2^{+}, 3^{-}, \ldots\right)$, while $D_{J}(3000)$ can be identified with unnatural parity states like $\left(0^{-}, 1^{+}, 2^{-}, 3^{+}, \ldots\right)$. Therefore, the missing doublets $2 S, 2 D, 1 F, 2 P$, and $3 S$ can be thought of as filled up with these states. We study the two-body strong decay widths and branching ratios of missing doublets and plot the branching ratios vs. the mass of the decaying particle. These plots are used to thoroughly analyze all assignments to $D_{J}(3000)$ and various possibilities for the $J^{P}$ values.
\end{abstract}

\section{Introduction}

The hadrons containing a single heavy quark can be analyzed in a framework which is formulated for $N_{f}$ heavy quarks with mass $m_{Q} \gg \Lambda_{\mathrm{QCD}}$ using heavy quark effective theory [3]. This theory assumes the heavy quark to act as a static color source and its spin is $s_{Q}$, which can be thought of as coupled to the spin of the light degrees of freedom $s_{l}$. Secondly, heavy quark flavor symmetry leads to interaction of the heavy

\footnotetext{
a e-mail: mbatra310@gmail.com

be-mails: alka.iisc@gmail.com; alka@thapar.edu
}

quark with the light degrees of freedom through exchange of soft gluons only. The heavy quark spin-flavor symmetry can be exploited further to explore several hadronic properties. The motivation for the present work arises due to the recently observed charm and bottom meson states by experimental collaborations like $\mathrm{BaBar}$ [1], $\mathrm{LHCb}$ [2] and CDF [4]. The heavy meson spectrum has recently aroused interest because of the various particles at different resonances and energies. Recently, some excited charm meson states were observed which are $D(2550), D(2600), D(2750)$, and $D(2760)$ in the decay channels $D^{0}(2550) \rightarrow D^{*+} \pi^{-}$, $D^{0}(2600) \rightarrow D^{*+} \pi^{-}, D^{+} \pi^{-}, D^{0}(2750) \rightarrow D^{+} \pi^{-}$, $D^{+}(2600) \rightarrow D^{0} \pi^{+}$, and $D^{+}(2760) \rightarrow D^{0} \pi^{+}$in the inclusive $e^{+} e^{-} \rightarrow c \bar{c}$ interactions by the BaBar Collaboration [1]. The most suitable spin-parity assignments for $D(2750), D(2760)$ is $\left(2^{-}, 3^{-}\right)$or $1 \mathrm{D}$ and for $D(2550)$, $D(2600)\left(0^{-}, 1^{-}\right)$, i.e. the $2 S$ state, respectively. The $\mathrm{LHCb}$ Collaboration [2] observed some new resonances in addition to the one above, i.e. $D_{J}(3000)^{+, 0}$ and $D_{J}^{*}(3000)^{0}$ around $3 \mathrm{GeV}$ in association with $D_{2}^{*}(2460)^{0}$ and $D_{J}^{*}(2760)^{0}$ and these exist in the $D^{+} \pi^{-}$invariant mass spectrum. The states $D_{J}^{*}(3000)^{+}, D_{2}^{*}(2460)^{+}$, and $D_{J}^{*}(2760)^{+}$were observed in the $D_{0} \pi^{+}$spectrum whereas the states measured in the $D^{*+} \pi^{-}$spectrum were $D_{1}(2420)^{0}, D_{2}^{*}(2460)^{0}, D_{J}^{*}(2760)^{0}$, $D_{J}(2580)^{0}, D_{J}(2740)^{0}$, and $D_{J}(3000)^{0}$, respectively. We have a similar case in the beauty sector. Very recently, the CDF Collaboration [4] has found evidence for a new resonance, $B(5970)$, simultaneously in the $B^{0} \pi^{+}$and $B^{+} \pi^{-}$ mass distributions with a significance of $4.4 \sigma$ standard deviations, and one further reported the first study of resonances with orbitally excited $B^{+}$mesons and an updated measurement of the orbitally excited $B^{0}$ and $B_{s}^{0}$ mesons. The branching ratio for $B_{s 2}^{* 0} \rightarrow B^{*+} K^{-}$decays is also measured. The masses of the new $B(5970)$ measured resonances are $5978 \pm 5$ (stat) \pm 12 (syst) $\mathrm{MeV} / \mathrm{c}^{2}$ for the neutral state and $5961 \pm 5$ (stat) \pm 12 (syst) $\mathrm{MeV} / \mathrm{c}^{2}$ for the charged state decaying into $B \pi$ states. This state is proposed as belong- 
ing to the radially excited bottom meson family. Therefore, in the past decades, we faced several ground states as well as excited states of the charm meson family, such as by the discovery of $D_{s J}(2460), D_{s J}(2632), D_{s J}(3040)$, etc. In the bottom meson family, we witness some new states such as $B(5279), B^{*}(5325)$ for the $n=1$ family in the $\left(0^{-}, 1^{-}\right)$ doublet. In the infinite heavy quark mass limit, a heavylight system $Q \bar{q}$ can be classified into doublets depending upon their quantum numbers. We have a heavy hadronic system containing a heavy quark with spin quantum number $S_{Q}$ and light degrees of freedom $s_{l}$ that includes light quark and gluons interacting through quark-antiquark pairs. It should have the quantum numbers of a light quark, that is, $S_{l}$, in order to have a total conserved quantum number $J$, where $J=S_{Q}+S_{l}$. Defining $J$ as $J^{2}=j(j+1)$ and $S_{Q}^{2}=\left(s_{Q}\right)\left(s_{Q}+1\right)$ and $S_{l}^{2}=\left(s_{l}\right)\left(s_{l}+1\right)$, the total spin $j \pm=s_{l} \pm \frac{1}{2}$ can be obtained by combining the spin of the heavy quark spin $\frac{1}{2}$ with the spin of the light degrees of freedom. The heavy mesonic system forms a degenerate doublet of a ground state with $J= \pm 1$ and a negative parity denoted $D$ and $D^{*}$ for the charm meson. The first excited states $0^{+}$ and $1^{+}$of the heavy mesons correspond to the quantum numbers of the $s_{l}^{p}=\frac{1}{2}^{+}$doublet. There is also an excited doublet of the heavy meson with $J^{P}=1^{+}$and $2^{+}$. Similarly, other excited mesonic states correspond to the $J^{P}$ states. In this article, we identify the recently observed charmed meson states $D_{J}(2550), D_{J}^{*}(2600), D_{J}(2740), D_{J}^{*}(2760)$, $D_{J}(3000)$, and $D_{J}^{*}(3000)$ by their $J^{P}$ assignment. These states were observed by the LHCb Collaboration and one predicted their decay widths and masses. We study the strong decays of these charmed mesons to the ground states of the heavy mesons along with the emission of pseudo-scalar pions in heavy quark effective theory in the leading order approximations. Although the same has been studied by [5], we extend that work by fitting the experimental data to find the coupling constants in various strong decays. Also, we include two additional possibilities for the assignment of the $J^{P}$ states to $D_{J}^{*}(3000)$ and $D_{J}(3000)$. In the end, we also try to justify all the possible assignments to $D_{J}^{*}(3000)$ and $D_{J}(3000)$ by analyzing their branching ratios graphically.

\section{The Lagrangian for strong decays to heavy mesons}

A single field $H_{a}$ annihilating the $s_{l}=\frac{1}{2}^{-}$meson doublet, pseudo-scalar, and vector mesons can be written as [6]

$H_{a}=\frac{1+\psi}{2}\left(P_{a}^{* \mu} \gamma_{\mu}-P_{a} \gamma_{5}\right)$.

Here $a$ is the SU(3) index. In the charm meson sector, $H_{a}$ consists of the $D^{0}, D^{+}, D_{s}^{+}$pseudo-scalar mesons and the
$D^{* 0}, D^{*+}, D_{s}^{*+}$ vector mesons. The lowest lying excited states are the $J^{P}=0^{+}$and $1^{+}$i.e. the $s_{l}^{P}=\frac{1}{2}^{+}$doublet represented by the fields $S_{a}$ [7]. The fields for the excited spin doublets are mentioned below:

$$
\begin{aligned}
S_{a}= & \frac{1+\psi}{2}\left(P_{1 a}^{\mu} \gamma_{\mu} \gamma_{5}-P_{0 a}^{*}\right), \\
T_{a}^{\mu}= & \frac{1+\psi}{2}\left(P_{2 a}^{* \mu \nu} \gamma_{\mu}-P_{1 a v} \sqrt{\frac{3}{2}} \gamma_{5}\right. \\
& \left.\times\left[g^{\mu \nu}-\frac{1}{3} \gamma^{\nu}\left(\gamma^{\mu}-v^{\mu}\right)\right]\right), \\
X_{a}^{\mu}= & \frac{1+\psi}{2}\left(P_{2 a}^{* \mu \nu} \gamma_{5} \gamma_{\nu}-P_{1 a \mu}^{*} \sqrt{\frac{3}{2}}\right. \\
& \left.\times\left[g^{\mu \nu}-\frac{1}{3} \gamma^{\nu}\left(\gamma^{\mu}+v^{\mu}\right)\right]\right), \\
Y_{a}^{\mu \nu}= & \frac{1+\psi}{2}\left(P_{3 a}^{* \mu \nu \sigma} \gamma_{\sigma}-P_{2 a}^{\alpha \beta} \sqrt{\frac{5}{3}} \gamma_{5}\right. \\
& \left.\times\left[g_{\alpha}^{\mu} g_{\beta}^{\nu}-\frac{g_{\beta}^{\nu} \gamma_{\alpha}\left(\gamma^{\mu}-v^{\mu}\right)}{5}-\frac{g_{\alpha}^{\mu} \gamma_{\beta}\left(\gamma^{\nu}-v^{\nu}\right)}{5}\right]\right), \\
Z_{a}^{\mu \nu}= & \frac{1+\psi}{2}\left(P_{3 a}^{* \mu \nu \sigma} \gamma_{5} \gamma_{\sigma}-P_{2 a}^{* \alpha \beta} \sqrt{\frac{5}{3}}\right. \\
& \left.\times\left[g_{\alpha}^{\mu} g_{\beta}^{\nu}-\frac{g_{\beta}^{\nu} \gamma_{\alpha}\left(\gamma^{\mu}+v^{\mu}\right)}{5}-\frac{g_{\alpha}^{\mu} \gamma_{\beta}\left(\gamma^{\nu}+v^{\nu}\right)}{5}\right]\right), \\
R_{a}^{\mu \nu \rho}= & \frac{1+\psi}{2}\left(P_{4 a}^{* \mu \nu \rho \sigma} \gamma_{5} \gamma_{\sigma}-P_{3 a}^{\alpha \beta \tau} \sqrt{\frac{7}{4}}\right. \\
& \times\left[g_{\alpha}^{\mu} g_{\beta}^{\nu} g_{\tau}^{\rho}-\frac{g_{\beta}^{\nu} g_{\tau}^{\rho} \gamma_{\alpha}\left(\gamma^{\mu}-v^{\mu}\right)}{7}\right. \\
& \left.\left.\frac{g_{\alpha}^{\nu} g_{\tau}^{\rho} \gamma_{\beta}\left(\gamma^{\mu}-v^{\mu}\right)}{7}-\frac{g_{\alpha}^{\mu} g_{\beta}^{\nu} \gamma_{\tau}\left(\gamma^{\rho}-v^{\rho}\right)}{7}\right]\right) \\
& (4) \\
& \\
5 &
\end{aligned}
$$

The super fields $H_{a}$ contain s-wave mesons whereas $S_{a}, T_{a}$ contain the p-wave mesons. The light pseudo-scalar mesons are described by the fields $\xi=\exp ^{\frac{i M}{f}}$. The pion octet is introduced by the vector and axial combinations $V^{\mu}=$ $\frac{1}{2} \xi \partial^{\mu} \xi^{\dagger}+\xi^{\dagger} \partial^{\mu} \xi$ and $A^{\mu}=\frac{1}{2} \xi \partial^{\mu} \xi^{\dagger}-\xi^{\dagger} \partial^{\mu} \xi$. We choose $f_{\pi}=130 \mathrm{MeV}$. Here, all traces are taken over the Dirac spinor indices, the light quark $\mathrm{SU}(3)_{V}$ flavor indices $a=$ $u, d, s$, and the heavy quark flavor indices $Q=c, b$. The Dirac structure of the chiral Lagrangian has been replaced by a velocity vector $v$. At the leading order, the heavy meson chiral Lagrangian terms $L_{H}, L_{S}, L_{T}, L_{X}, L_{Y}, L_{Z}, L_{R}$ for the strong decays to the $D^{(*)} \pi, D^{(*)} \eta$ and $D_{s}^{(*)} K$ states can be written as 


$$
\begin{aligned}
L_{H}= & g_{H} \operatorname{Tr}\left\{\bar{H}_{a} H_{b} \gamma_{\mu} \gamma_{5} A_{b a}^{\mu}\right\}, \\
L_{S}= & g_{S} \operatorname{Tr}\left\{\bar{H}_{a} S_{b} \gamma_{\mu} \gamma_{5} A_{b a}^{\mu}\right\}+\text { h.c., } \\
L_{T}= & \frac{g_{T}}{\Lambda} \operatorname{Tr}\left(\bar{H}_{a} T_{b}^{\mu}\left(D_{\mu} A+i D A_{\mu}\right)_{b a} \gamma_{5}\right)+\text { h.c., } \\
L_{X}= & \frac{g_{X}}{\Lambda} \operatorname{Tr}\left(H_{a} X_{b}^{\mu}\left(i D_{\mu} A+i D A_{\mu}\right)_{b a} \gamma_{5}\right)+\text { h.c., } \\
L_{Y}= & \frac{1}{\Lambda^{2}} \operatorname{Tr}\left(\overline { H } _ { a } Y _ { b } ^ { \mu \nu } \left[k_{1}^{Y}\left\{D_{\mu}, D_{v}\right\} A_{\lambda}\right.\right. \\
& \left.\left.+k_{2}^{Y}\left(D_{\mu} D_{\lambda} A_{\mu}+D_{\nu} D_{\lambda} A_{\mu}\right)\right]_{b a} \gamma^{\lambda} \gamma_{5}\right)+ \text { h.c., } \\
L_{Z}= & \frac{1}{\Lambda^{2}} \operatorname{Tr}\left(\bar{H}_{a} Z_{b}^{\mu \nu}\left[k_{1}^{z}\left\{D_{\mu}, D_{v}\right\}, D_{v}\right\} A_{\lambda}\right. \\
& \left.\left.+k_{2}^{Z}\left(D_{\mu}, D_{\lambda} A_{v}+D_{v} D_{\lambda} A_{\mu}\right)\right]_{b a} \gamma^{\lambda} \gamma_{5}\right)+ \text { h.c., }
\end{aligned}
$$

where $D_{\mu}=\partial_{\mu}+v_{\mu},\left\{D_{\mu}, D_{\nu}\right\}=D_{\mu} D_{\nu}+D_{\mu} D_{\nu}$, $\left\{D_{\mu}, D_{\nu}, D_{\rho}\right\}=D_{\mu} D_{v} D_{\rho}+D_{\mu} D_{\rho} D_{v}+D_{\nu} D_{\rho} D_{\mu}+$ $D_{\rho} D_{\mu} D_{v}+D_{\rho} D_{\nu} D_{\mu}$. These terms describe the transitions of positive and negative parity mesons with the emission of light pseudo-scalar mesons. The mixing angles between two states are determined by including a spin symmetry violating correction in the Lagrangian. The term should respect parity and time reversal and it may be of the generic form as written thus:

$L_{d 1}=\frac{h_{1}}{2 m \Lambda} \operatorname{Tr}\left[\bar{H} \sigma^{\mu \nu} T^{\alpha} \sigma_{\mu \nu} \gamma^{k} \gamma^{5}\left(i D_{\alpha} A_{\kappa}+i D_{\kappa} A_{\alpha}\right)\right]+$ h.c.

The corresponding operator for the mixing of $1^{+}$in $2 S$ and $1 D$, respectively, is due to the spin symmetry violating effect and can be written as $L_{\text {mix }}=g_{1} \operatorname{Tr}\left[\overline{\tilde{H}} \phi_{s}^{\mu \nu} X_{\mu} \sigma_{\nu \alpha} v^{\alpha}\right]+$ h.c.

\section{Strong decay width formula and coupling constants}

From the chiral Lagrangian terms, we can obtain the decay widths $\Gamma$ for the strong decays to the final states $D^{(*)} \pi$, $D^{(*)} \eta, D_{s}^{(*)} K$ where the symmetry breaking scale $\Lambda_{X}=$ $1 \mathrm{GeV}$. We now write the expression for the decay widths; we consider various doublets, which the decaying meson belongs to, to be as follows, where $M$ denotes the emission of pseudo-scalar mesons, i.e. the $\pi, K$, and $\eta$ fields: $\left(0^{-}, 1^{-}\right)$to $\left(0^{-}, 1^{-}\right)+M$ :

$\Gamma\left(1^{-} \rightarrow 0^{-}\right)=C_{M} \frac{g_{H}^{2}}{6 \pi f_{\pi}^{2}} \frac{M_{f}}{M_{i}}\left|\overrightarrow{p_{M}}\right|^{3}$,
$\Gamma\left(1^{-} \rightarrow 1^{-}\right)=C_{M} \frac{g_{H}^{2}}{3 \pi f_{\pi}^{2}} \frac{M_{f}}{M_{i}}\left|\overrightarrow{p_{M}}\right|^{3}$,

$\left(0^{+}, 1^{+}\right)$to $\left(0^{-}, 1^{-}\right)+M$ :

$\Gamma\left(1^{+} \rightarrow 1^{-}\right)=C_{M} \frac{g_{S}^{2}}{2 \pi f_{\pi}^{2}} \frac{M_{f}}{M_{i}}\left|\overrightarrow{p_{M}}\right|\left[m_{M}^{2}+\left|\overrightarrow{p_{M}}\right|^{2}\right]$,

$\Gamma\left(1^{+} \rightarrow 0^{-}\right)=C_{M} \frac{g_{S}^{2}}{2 \pi f_{\pi}^{2}} \frac{M_{f}}{M_{i}}\left|\overrightarrow{p_{M}}\right|\left[m_{M}^{2}+\left|\overrightarrow{p_{M}}\right|^{2}\right.$, $\left(1^{+}, 2^{+}\right)$to $\left(0^{-}, 1^{-}\right)+M$ :

$\Gamma\left(1^{+} \rightarrow 1^{-}\right)=C_{M} \frac{2 g_{T}^{2}}{3 \pi f_{\pi}^{2} \Lambda^{2}} \frac{M_{f}}{M_{i}}\left|\vec{p}_{M}^{5}\right|$,

$\Gamma\left(2^{+} \rightarrow 0^{-}\right)=C_{M} \frac{4 g_{T}^{2}}{15 \pi f_{\pi}^{2} \Lambda^{2}} \frac{M_{f}}{M_{i}}\left|\overrightarrow{p_{M}}\right|$,

$\Gamma\left(2^{+} \rightarrow 1^{-}\right)=C_{M} \frac{2 g_{T}^{2}}{5 \pi f_{\pi}^{2} \Lambda^{2}} \frac{M_{f}}{M_{i}}\left|\vec{p}_{M}{ }^{5}\right|$,

$\left(1^{-}, 2^{-}\right)$to $\left(0^{-}, 1^{-}\right)+M$ :

$\Gamma\left(1^{-} \rightarrow 0^{-}\right)=C_{M} \frac{4 g_{X}^{2}}{9 \pi f_{\pi}^{2} \Lambda^{2}} \frac{M_{f}}{M_{i}}\left|\overrightarrow{p_{M}}{ }^{3}\right|\left[m_{M}^{2}+\left|\overrightarrow{p_{M}}\right|^{2}\right]$,

$\Gamma\left(1^{-} \rightarrow 1^{-}\right)=C_{M} \frac{2 g_{X}^{2}}{9 \pi f_{\pi}^{2} \Lambda^{2}} \frac{M_{f}}{M_{i}}\left|\vec{p}_{M}^{3}\right|\left[m_{M}^{2}+\left|\overrightarrow{p_{M}}\right|^{2}\right]$,

$\Gamma\left(2^{-} \rightarrow 1^{-}\right)=C_{M} \frac{2 g_{X}^{2}}{3 \pi f_{\pi}^{2} \Lambda^{2}} \frac{M_{f}}{M_{i}}\left|\vec{p}_{M}^{5}\right|\left[m_{M}^{2}+\left|\overrightarrow{p_{M}}\right|^{2}\right]$,

$\left(2^{-}, 3^{-}\right)$to $\left(0^{-}, 1^{-}\right)+M$ :

$\Gamma\left(2^{-} \rightarrow 1^{-}\right)=C_{M} \frac{4 g_{Y}^{2}}{15 \pi f_{\pi}^{2} \Lambda^{4}} \frac{M_{f}}{M_{i}}\left|\overrightarrow{p_{M}}\right|$,

$\Gamma\left(3^{-} \rightarrow 0^{-}\right)=C_{M} \frac{4 g_{Y}^{2}}{35 \pi f_{\pi}^{2} \Lambda^{4}} \frac{M_{f}}{M_{i}}\left|\vec{p}_{M}{ }^{7}\right|$,

$\Gamma\left(3^{-} \rightarrow 1^{-}\right)=C_{M} \frac{16 g_{Y}^{2}}{105 \pi f_{\pi}^{2} \Lambda^{4}} \frac{M_{f}}{M_{i}}\left|\overrightarrow{p_{M}}{ }^{7}\right|$.

The coefficients $C_{M}$ are different for the various light pseudoscalar mesons: $C_{\pi^{+}}=C_{K^{+}}=1, C_{\pi^{0}}=C_{K_{s}}=\frac{1}{2}, C_{\eta}=\frac{1}{6}$. $\overrightarrow{p_{M}}$ is the three momentum of M. The higher order corrections to the heavy quark limit can also be considered by adding terms of the order $\frac{1}{m_{Q}}$ with some unknown constants. The decay rates depend upon the effective coupling constants. The parameters used in the above expressions for the decay widths are taken from the particle data group [8]. Thus the numerical values of the decay widths come to us in terms of the coupling constants $g_{H}, g_{Y}, g_{X}$, etc. Here the first radial excitation of $D^{*}$ is represented as $\tilde{D}^{*}$. The first radially excited state of $\mathrm{H}$ is governed by the decay constant $\tilde{g_{H}}$, which can be fitted to the experimental data within the mass range of 2600-2700 MeV. The coupling constants can either be determined theoretically or by fitting the experimental data. However, various quark models $[9,10]$ and sum rule (e.g. QCD sum rules) [11-13] techniques predict the coupling constants. Another possible method is to use lattice QCD [14] which incorporates QCD as a first principle. Using the experimental data of the decay widths and branching ratios as input, one can fit the experimental data to find 
Table 1 Experimental status of the latest non-strange charm meson states and their masses and decay widths by BaBar and LHCb Collaborations

\begin{tabular}{lllllll}
\hline Sr. no & Charm meson state & Mass $(\mathrm{MeV})[\mathrm{LHCb}]$ & Mass $(\mathrm{MeV})[\mathrm{BaBar}]$ & Width $(\mathrm{MeV})[\mathrm{LHCb}]$ & Width $(\mathrm{MeV})[\mathrm{BaBar}]$ & Decay channel \\
\hline 1 & $D_{J}^{*}(2650)^{0}$ & $2649.2 \pm 3.5 \pm 3.5$ & $2608.7 \pm 2.4 \pm 2.5$ & $140.2 \pm 17.1 \pm 18.6$ & $93 \pm 6 \pm 13$ & $D^{*+} \pi^{-}$ \\
2 & $D_{J}^{*}(2760)^{0}$ & $2761.1 \pm 5.1 \pm 6.5$ & $2763.3 \pm 2.3 \pm 2.3$ & $74.4 \pm 3.4 \pm 37.0$ & $60.9 \pm 5.1 \pm 3.6$ & $D^{*+} \pi^{-}$ \\
3 & $D_{J}(2580)^{0}$ & $2579.5 \pm 3.4 \pm 5.5$ & $2539.4 \pm 4.5 \pm 6.8$ & $177.5 \pm 17.8 \pm 46.0$ & $130 \pm 12 \pm 13$ & $D^{*+} \pi^{-}$ \\
4 & $D_{J}(2740)^{0}$ & $2737.0 \pm 3.5 \pm 11.2$ & $2752.4 \pm 1.7 \pm 2.7$ & $73.2 \pm 13.4 \pm 25.0$ & $71 \pm 6 \pm 11$ & $D^{*+} \pi^{-}$ \\
5 & $D_{J}(3000)^{0}$ & $2971.8 \pm 8.7$ & & $188.1 \pm 44.8$ & $D^{*+} \pi^{-}$ \\
6 & $D_{J}^{*}(2760)^{0}$ & $2760.1 \pm 1.1 \pm 3.7$ & & $74.4 \pm 3.4 \pm 19.1$ & $D^{+} \pi^{-}$ \\
7 & $D_{J}^{*}(3000)^{0}$ & $3008.1 \pm 4.0$ & & $110.5 \pm 11.5$ & $D^{+} \pi^{-}$ \\
8 & $D_{J}^{*}(2760)^{+}$ & $2771.7 \pm 1.7 \pm 3.8$ & $2769.7 \pm 3.8 \pm 1.5$ & $66.7 \pm 6.6 \pm 10.5$ & 60.9 & $D^{0} \pi^{+}$ \\
9 & $D_{J}^{*}(3000)^{+}$ & 3008.1 & 110.5 & $D^{0} \pi^{+}$ \\
\hline
\end{tabular}

the effective coupling constants. The coupling constants play an important role in heavy quark phenomenology. They are directly related to the charm meson strong decays and are further useful to explore other decays of charm mesons involving pionic emissions.

\section{Spin-parity analysis for non-strange charm meson states}

The recent experimental data of charm meson states from $\mathrm{LHCb}$ and $\mathrm{BaBar}$ Collaboration motivates us to find the best fit values of coupling constants in strong decays. Table 1 presents the recent experimental data of the non-strange charm mesons.

The states with $J^{P}=\left(0^{-}, 1^{-}, 0^{+}, 1^{+}, 2^{+}\right)$are well known. The doublets having spin-parity assignments $s_{l}^{P}=$ $\frac{3}{2}^{+}$consist of $D_{1}(2420)$ and $D_{2}^{*}(2460)$ in the non-strange sector. The states $D_{0}^{*}(2400), D_{1}^{\prime}(2430)$ belong to the $s_{l}^{P}=$ $\frac{1}{2}^{+}$charm doublet [1]. The experimental data on the decay widths suggest that the states $\left(0^{+}, 1^{+}\right)$are quite broad, expected to decay via the s-wave, whereas the states belonging to $\left(1^{+}, 2^{+}\right)$doublets are quite narrow and decay via the $\mathrm{d}$-wave. The branching ratio measured by the BaBar Collaboration is given by

$$
\frac{\operatorname{BR}\left(D_{2}^{* 0}(2460) \rightarrow D^{+} \pi^{-}\right)}{\operatorname{BR}\left(D_{2}^{* 0}(2460) \rightarrow D^{*+} \pi^{-}\right)}=1.47 \pm 0.03 \pm 0.16
$$

There are few more recent states whose branching ratios as measured by BaBar are

$$
\begin{aligned}
& \frac{\operatorname{BR}\left(D^{0}(2600) \rightarrow D^{+} \pi^{-}\right)}{\operatorname{BR}\left(D^{0}(2600) \rightarrow D^{*+} \pi^{-}\right)}=0.32 \pm 0.02 \pm 0.09 \\
& \frac{\operatorname{BR}\left(D^{0}(2760) \rightarrow D^{+} \pi^{-}\right)}{\operatorname{BR}\left(D^{0}(2750) \rightarrow D^{*+} \pi^{-}\right)}=0.42 \pm 0.05 \pm 0.11
\end{aligned}
$$

The information from the BaBar Collaboration and the quark model suggests that the $D^{0}(2550)$ state corresponds to the $0^{-}$state. The $D^{0}(2600)$ corresponds to the $1^{-}$state either in the $2 S$ or $1 D$ spectrum, respectively, because this state was observed in both the $D \pi$ and the $D^{*} \pi$ channels. If we find the mass of these particular states using heavy quark symmetry and other theoretical models $[15,16]$, it can be suggested that the state $D(2600)$ can be identified as either a radial excitation of the heavy quark doublet $H$ or as $1 \mathrm{D}$. The branching ratios for $D \pi$ and $D^{*} \pi$ for both decay states are calculated as $\frac{\mathrm{BR}\left(D^{0}(2600) \rightarrow D^{+} \pi^{-}\right)}{\operatorname{BR}\left(D^{0}(2600) \rightarrow D^{*+} \pi^{-}\right)}=0.82$ for $2 S$ and $\frac{\operatorname{BR}\left(D^{0}(2600) \rightarrow D^{+} \pi^{-}\right)}{\operatorname{BR}\left(D^{0}(2600) \rightarrow D^{*+} \pi^{-}\right)}=0.38$ for $1 \mathrm{D}$, respectively. The comparison with the experimental data results in favor of the $1 \mathrm{D}$ assignment. The theoretical estimation of the coupling constant for the strong decay width of the mesons in this particular state is $0.53 \pm 0.01$. The theoretical estimation of the branching ratios from the heavy quark effective theory [17] leads to the conclusion that there may be violations in the flavor and the spin symmetry. In Ref. [18], Sun et al. used the ${ }^{3} P_{0}$ model to examine the strong decays of these states and they concluded that the $D^{0}(2600)$ state can be identified as a mixture of the $2^{3} S_{1}$ and $1^{3} D_{1}$ states. Therefore, the other possibility is that $D(2600)$ may be considered as a mixing state of $2 S$ and $1 D$, respectively. The other two states $D(2750)$ and $D(2760)$ can be identified by the $J^{P}=$ $\left(2^{-}, 3^{-}\right)$assignment. It is very interesting to point out that a non-strange partner of $D_{S J}(2860)$ can be associated with $D$ (2760) due to the mass gap, which is about $150 \mathrm{MeV}$. There are also several references like $[5,17]$ which suggest a possible assignment for $D(2750)$ and $D^{*}(2760)$ state with the $l=2, n=1$ state. Moreover, the branching ratio measurement $\frac{\operatorname{BR}\left(D^{0}(2760) \rightarrow D^{+} \pi^{-}\right)}{\operatorname{BR}\left(D^{0}(2750) \rightarrow D^{*+} \pi^{-}\right)}$gives the value of 0.80 from the leading order effective theory, which is found to match with the experimental data. Saturating the total decay width with the ground state to two-body decays, we can fit the experimental data of $\mathrm{LHCb}$ and $\mathrm{BaBar}$ to estimate the coupling constant. We take the experimental data of the decay widths of recent states as presented in Table 1 to find the hadronic coupling constants. The decay widths are calculated using the 
decay formulas given above. We can fit the experimental data of the BaBar and LHCb Collaborations to estimate the coupling constants. The observed radially excited non-strange charm meson states in the heavy meson spectrum are the two resonances $\left(D(2550), D^{*}(2600)\right)$. The value of the coupling constant is obtained from the measured width of $(D(2550))$ and the computed value is $0.35 \pm 0.03$ [17]. The calculated value of the coupling constants in our fitting program for $D$ (2550) turns out to be $0.40 \pm 0.05$. The best fit value of these coupling constants is estimated within experimental error using the chi-square minimization technique. The errors are clearly dominated by the statistical and systematic uncertainties. To check the consistency of our fitting algorithm, the coupling constant estimation was carried out for the decays of $J^{P}=\left(0^{+}, 1^{+}\right)$and the fitted value turns out to be $0.53 \pm 0.04$. This value has been found to match well with predictions from other theoretical approaches [17]. The value of the coupling constants for the $D(2750)$ and $D^{*}(2760)$ states are also estimated in our fitting program; they are $0.61 \pm 0.01$ and $0.79 \pm 0.03$. Let us consider the new states observed by LHCb in the $D \pi$ and $D^{*} \pi$ spectrum; from the strong decays, the states are labeled as $D_{J}^{*}(3000)^{0} \rightarrow D^{+} \pi^{-}$and $D_{J}^{*}(3000)^{+} \rightarrow D^{0} \pi^{+}$. On the basis of LHCb data [1], the angular distribution of $D_{J}(3000) \rightarrow D^{*} \pi$ is found to be consistent with an unnatural parity. The possible spin-parity assignment for $D_{J}(3000)^{0}$ can be $J^{P}=0^{-}, 1^{+}, 2^{-}, 3^{+}, \ldots$, and $D_{J}^{*}(3000)$ has the possible spin-parity assignments $J^{P}=0^{+}, 1^{-}, 2^{+}, 3^{-}, 4^{+}, \ldots$ Thus, it can be stated that the two states can be higher radial excitations or can belong to $1 F$ states in the meson spectrum. Some possible indications about the possible assignment of $J^{P}$ quantum numbers can be realized from the masses of these states. One of the well-known potential models [15] calculated the masses of all possible excited mesonic states and we can suggest the possible spin and parities assignments of these newly discovered states. To extract detailed information as regards the newly observed states, we present a summary of all the $D$ meson states and various possibilities of $D_{J}^{*}(3000)$ and $D_{J}^{*}(3000)^{0}$ in Table 2.

Wang [5] also suggested various possibilities for the $D_{J}^{*}(3000)$ states and calculated the decay widths of these states in terms of the relevant coupling constants. We also suggest the same but we add two more possibilities, i.e. $\left(1^{-}, 2^{-}\right)$and $\left(2^{-}, 3^{-}\right)$lying in the $1 D$ spectrum. To analyze the spectrum of the above $J^{P}$ states, we study the two-body decay behavior and calculate the branching ratios for the states for which the decay to $P M$ and $P^{*} M$ both are allowed. Considering the branching ratios for the strong decays $\frac{\operatorname{BR}\left(D_{2}^{*} \rightarrow D^{*+} \pi^{-}\right)}{\operatorname{BR}\left(D_{2}^{*} \rightarrow D^{+} \pi^{-}\right)}$, the decay occurs via relative f-wave and similarly for the other decay channels; the branching ratio and the relative wave are presented in Table 3.

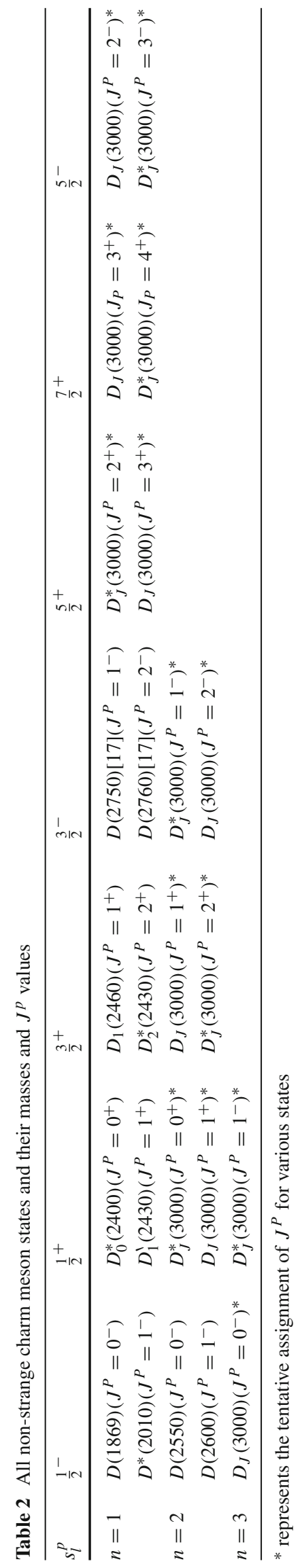


Table 3 Ratios of decay widths for $D_{J}^{*}(3000)$ state in all possible assignments. The relative wave emitted in the decay is also presented for all the possible assignments

\begin{tabular}{lll}
\hline$D_{J}^{*}(3000)$ & $D_{J}^{*}(3000) \rightarrow D^{*} \pi$ & $\frac{\mathrm{BR}\left(D_{J}^{*} \rightarrow D^{*+} \pi^{-}\right)}{\mathrm{BR}\left(D_{J}^{*} \rightarrow D^{+} \pi^{-}\right)}$ \\
\hline$s_{l}^{p}=\frac{5}{2}^{+}, J^{P}=2^{+}$ & f-wave & 0.343 \\
$s_{l}^{p}=\frac{7}{2}^{+}, J^{P}=4^{+}$ & f-wave & 0.52 \\
$s_{l}^{p}=\frac{1}{2}^{+}, J^{P}=0^{+}$ & s-wave & 0 \\
$s_{l}^{p}=\frac{3}{2}^{+}, J^{P}=2^{+}$ & d-wave & 0.955 \\
$s_{l}^{p}=\frac{1}{2}^{-}, J^{P}=1^{-}$ & p-wave & 1.57 \\
$s_{l}^{p}=\frac{5}{2}^{-}, J^{P}=3^{-}$ & f-wave & 0.68 \\
$s_{l}^{p}=\frac{3}{2}^{-}, J^{P}=1^{-}$ & p-wave & 0.32 \\
\hline
\end{tabular}

Table 3 collects the ratios of the decay widths for the $D_{J}^{*}(3000)$ state. The predicted ratios along with the graphs can be analyzed to exclude some of the assignments.
The graphs show the variation of the branching ratios vs. the mass of decaying particle (Fig. 1). From the graphs, it can be stated that the states lying in the $3 S$ doublet produce ratios of more than one around the mass values of $3000 \mathrm{MeV}$. The decay of $D_{J}^{*}(3000)$ into $D^{*+} \pi^{-}$is the dominant mode for the $3 S$ doublet, which is inconsistent with the experimental observations. $D_{J}(3000)$ is observed to decay via the $D^{+} \pi^{-}$ channel. However, if we assume the $D_{J}(3000)$ as the spin partner of $D_{J}^{*}(3000)$ then $D^{*} \pi$ is the most dominant decay mode. The other possible decay modes are $D K$ and $D \eta$. If $D_{J}(3000)$ and $D_{J}^{*}(3000)$ belong to the $2 P\left(0^{+}, 1^{+}\right)$doublet then the allowed decay mode for $D_{J}^{*}(3000)$ forbids $D^{*+} \pi^{-}$ decay channels; this agrees well with the experimental observation. In all other cases, decays to both channels $D \pi$ and $D^{*} \pi$ are allowed. Moreover, the theoretical calculation of the branching ratios $\left(\frac{D_{J}^{*}(3000) \rightarrow D^{+} \pi^{-}}{D_{J}^{*}(3000) \rightarrow D^{*+} \pi^{-}}\right)$for all possible doublets and a comparison with the mass spectrum observed by the LHCb Collaboration suggest that the possible assignment of these two states can be either $\left(J^{P}=\left(1^{-}, 2^{-}\right)\right)$or
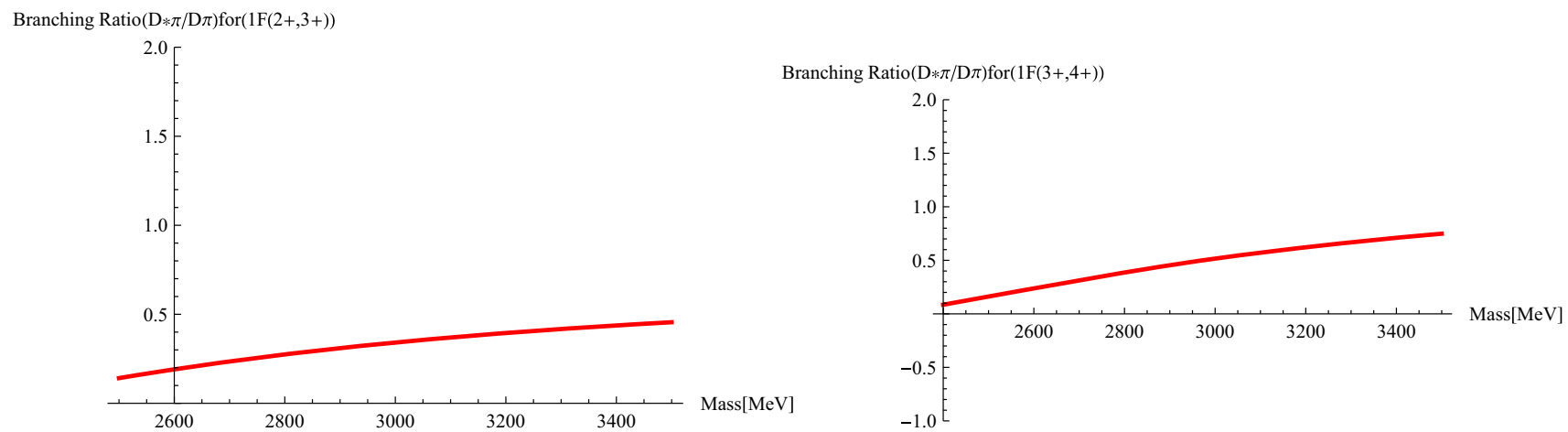

Branching Ratio(D $* \pi / \mathrm{D} \pi)$ for $(2 \mathrm{P})$

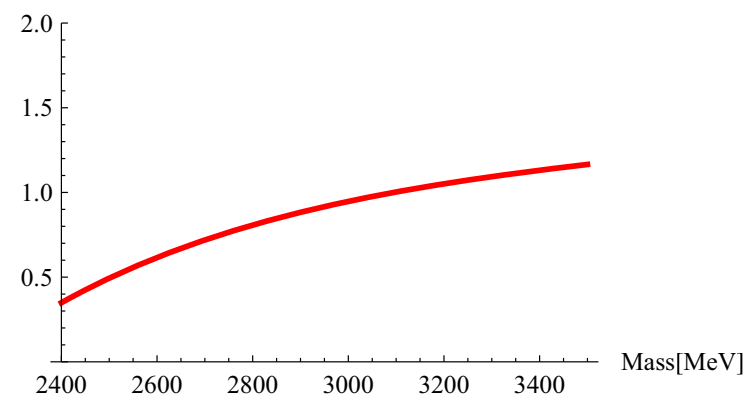

Branching Ratio $(\mathrm{D} * \pi / \mathrm{D} \pi)$ for $(2 \mathrm{D}(1-, 2-))$

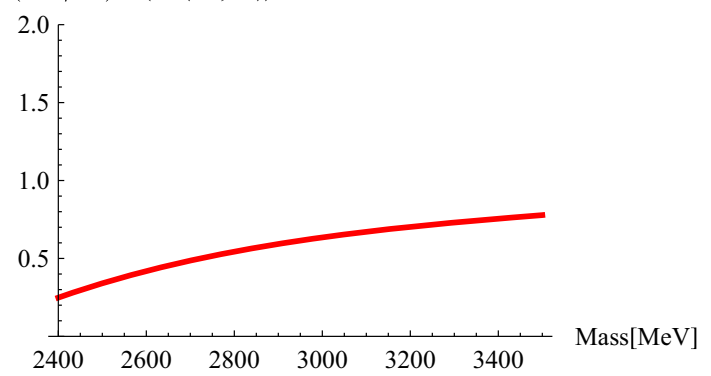

Branching Ratio(D $* \pi / \mathrm{D} \pi)$ for $(3 \mathrm{~S})$

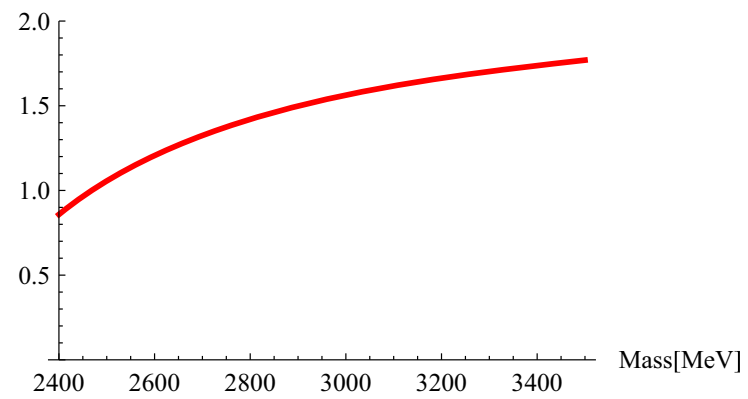

Branching Ratio $(\mathrm{D} * \pi / \mathrm{D} \pi)$ for $(2 \mathrm{D}(2-, 3-))$

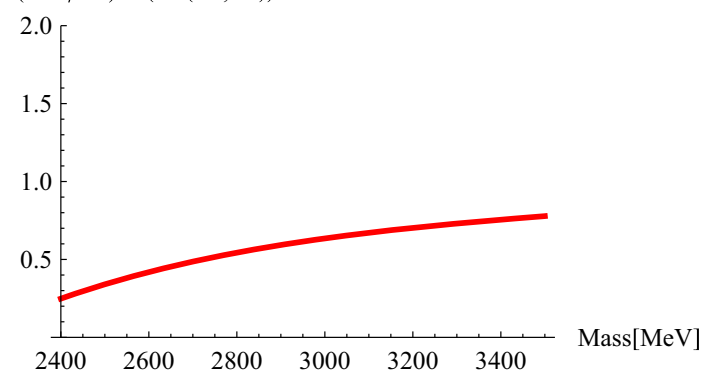

Fig. 1 Graphs showing branching ratios $\left(\frac{\mathrm{BR}\left(D_{J}^{*} \rightarrow D^{*+} \pi^{-}\right)}{\mathrm{BR}\left(D_{J}^{*} \rightarrow D^{+} \pi^{-}\right)}\right)$vs. mass of decaying particle 
$\left(J^{P}=\left(2^{+}, 3^{+}\right)\right)$for $D_{J}^{*}(3000)$ and $D_{J}(3000)$ The state $3^{3} S_{1}$ state decays to $D^{*} \pi, D \pi, D_{s}^{*} K, D_{s} K$, and $D \eta$, and the coupling constant for this particular state lies near the value of $\simeq 0.1$. Also, the state $3^{3} S_{1}$ appears to be a narrow $D$ meson state. But its partial decay width to $D^{*} \pi$ appears to be greater than that of the $D \pi$ mode, which is completely inconsistent with observations by the LHCb Collaboration. If we assign the $2 P\left(J^{P}=\left(1^{+}, 2^{+}\right)\right)$doublet to the $D_{J}^{*}(3000)$ and $D_{J}(3000)$ mesons, then the coupling constant for the strong decays to $\pi, K, \eta$ should lie between 0.12 and 0.15 . For the $\left(2^{3} D_{3}\right)$ state, $(D \pi, D K, D \eta)$ are the other allowed decay modes. Additional decay channels may include $D(2460) \pi$ and $D(2420) \pi$. The additional information as regards these decay channels may help to estimate the coupling constant more precisely. In addition to this, for the $2^{3} D_{1}$ state, the most prominent decay mode is $D \pi$; therefore the ideal decay mode to search for this state is $D \pi$. The ratio of the total decay widths for $D_{J}^{*}(3000)$ and $D_{J}(3000)$ can be calculated from the experimental data of the LHCb Collaboration. We have

$\frac{\Gamma\left(D_{J}^{*}(3000)\right)}{\Gamma\left(D_{J}(3000)\right)}=0.587 \pm 0.083$.

In our work, $\frac{\Gamma\left(D_{J}^{*}(3000)\right)}{\Gamma\left(D_{J}(3000)\right)}$ is calculated in heavy quark effective theory. The comparison of the theoretical value with the experimental data favors an $\left(1^{-}, 2^{-}\right)$assignment. A similar analysis can be carried out for the $D_{J}(3000)$ mesons. The various possibilities include $3{ }^{1} S_{0}, 2 P\left(1^{+}\right)$, and $2 D\left(2^{-}\right)$and $1 F$, etc. A closer look at the decay width of all the above states in the $D^{*} \pi$ spectrum suggests that the best possible assignment for $D_{J}^{*}(3000)$ and $D_{J}(3000)$ belongs to either the $2 P$ or $2 D$ state. The most prominent decay mode for $2 P\left(1^{+}\right)$in the $\left(1^{+}, 2^{+}\right)$doublet is found to be $D^{*} \pi$, which matches well with the experimental data on the decay width for $D_{J}(3000)$. However, if we consider the $D_{J}(3000)$ as the spin partner of $D_{J}^{*}(3000)$, then another possibility might be that $2 P\left(1^{+}\right)$in $\left(0^{+}, 1^{+}\right)$overlaps with that with $1^{+}$in the $\left(1^{+}, 2^{+}\right)$doublet.

\section{Conclusion}

We study the heavy meson decay width in the framework of heavy quark effective theory, which represents heavy quark and chiral symmetry at the chiral symmetry breaking scale $\Lambda_{\chi} \simeq 1 \mathrm{GeV}$ [7]. We studied the recent charm meson states with their $J^{p}$ assignment. The upcoming results at collaborations like $\mathrm{LHCb}$ produces the data for branching ratios that is used to calculate the decay width, coupling constants, and suitable $J^{p}$ states. The coupling constants and their studies are important to study heavy meson phenomenology. The accurate estimation of the coupling constants helps to study the detailed interaction of the heavy mesons. The present work calculates the coupling constant for the strong decays of the non-strange charm meson states ( $D(2550), D(2600), D(2750)$, and $D(2760)$ ) by using chisquare minimization techniques. The numerical values of the decay widths from collaborations like $\mathrm{LHCb}, \mathrm{BaBaR}$, and $\mathrm{CDF}$ are used to extract the values of the coupling constants. The various assignments of the $J^{p}$ values to the above mentioned states are also analyzed. The $J^{p}$ assignment for the $D^{0}(2550)$ state is $0^{-}$, while $D^{0}(2600)$ is identified as a mixture of the $2^{3} S_{1}$ and $1^{3} D_{1}$ states with $J^{p}=1^{-}$. The states $D(2750)$ and $D(2760)$ are identified by the $J^{P}=\left(2^{-}, 3^{-}\right)$ assignment. All assignments to $D_{J}(3000)$ are analyzed thoroughly and various possibilities for $J^{P}$ states have been checked. Two more possibilities, i.e. $\left(1^{-}, 2^{-}\right)$and $\left(2^{-}, 3^{-}\right)$ lying in the $2 D$ spectrum, have also been included for analysis. The best possible assignment in the present work favored $2 P\left(1^{+}\right)$for the $D_{J}(3000)$ state. While investigating the decays, it is concluded that the results on the decay widths are further helpful to search for unknown resonances, so that the excited meson spectrum for the $D$ meson family becomes clear to theorists as well as experimentalists.

Acknowledgments Part of the work is done under UGC Major Research Project No. SR/41-959/2012.

Open Access This article is distributed under the terms of the Creative Commons Attribution 4.0 International License (http://creativecomm ons.org/licenses/by/4.0/), which permits unrestricted use, distribution, and reproduction in any medium, provided you give appropriate credit to the original author(s) and the source, provide a link to the Creative Commons license, and indicate if changes were made.

Funded by $\mathrm{SCOAP}^{3}$.

\section{References}

1. P. del Amo Sanchez et al., Phys. Rev. D 82, 111101 (2010)

2. R. Aaij et al. [LHCb Collaboration], J. High Energy Phys. 1309, 145 (2013)

3. M. Neubert, Phys. Rep. 245, 259 (1994)

4. T.A. Aaltonen et al. [CDF Collaboration], Phys. Rev. D 90, 012013 (2014)

5. Z. Wang, Phys. Rev. D 88, 114003 (2013)

6. A.F. Falk, Phys. Lett. B 292, 119 (1992)

7. A.F. Falk, T. Mehan, Phys. Rev. D 53, 231 (1996)

8. J. Beringer et al. [Particle Data Group], Phys. Rev. D 86, 010001 (2012)

9. P.J. O’Donnell, Q.P. Xu, Phys. Lett. B 336, 113 (1994)

10. D. Becirevic, A.L. Yaouanc, J. High Energy Phys. 3, 021 (1999)

11. P. Colangelo, F.D. Fazio, G. Nardulli, Phys. Lett. B 334, 175 (1994)

12. P. Colangelo, F.D. Fazio, Eur. Phys. J. C 4, 503 (1998)

13. A.G. Grozin, O.I. Yakovlev, Eur. Phys. J. C 20, 723 (1998)

14. G.M. de Divittis et al. [UKQCD Collaboration], J High Energy Phys. 10, 10 (1998)

15. D. Ebert, R.N. Faustav, V.O. Galkin, Eur. Phys. J. C 66, 197 (2010)

16. M. Di Pierro, E. Eichten, Phys. Rev. D 64, 114004 (2001)

17. P. Colangelo, F.D. Fazio, R. Ferrandes, Phys. Rev. D 86, 054024 (2012)

18. Z.F. Sun, J.S. Yu, X. Liu, Phys. Rev. D 82, 111501 (2010) 